\title{
High-efficiency free-form nonimaging condenser overcoming rotational symmetry limitations
}

Juan Miñano, Pablo Benítez, José Blen, Asunción Santamaría

Juan C. Miñano, Pablo Benítez, José Blen, Asunción Santamaría, "Highefficiency free-form nonimaging condenser overcoming rotational symmetry limitations," Proc. SPIE 7103, Illumination Optics, 71030A (23 September 2008); doi: 10.1117/12.794259

SPIE. Event: Optical Systems Design, 2008, Glasgow, Scotland, United Kingdom 


\title{
High-efficiency free-form nonimaging condenser overcoming rotational symmetry limitations
}

\author{
Juan C. Miñano ${ }^{* a b}$, Pablo Benítez ${ }^{\mathrm{ab}}$, José Blen ${ }^{\mathrm{b}}$, Asunción Santamaría ${ }^{\mathrm{a}}$ \\ ${ }^{a}$ Universidad Politécnica de Madrid, Cedint, Campus Montegancedo, Pozuelo, 28223 Madrid, Spain; \\ bPI, 2400 Lincoln Avenue, Altadena, CA 91001, USA
}

\begin{abstract}
The importance of condenser optics is the fact that it is the bottleneck limiting efficiency in commercially available projection systems. Conventional condensers use rotational symmetric devices, most of them being elliptic or parabolic mirrors. They perform very far from the theoretical limits for sources such as arc lamps or halogen bulbs. Typical small displays in the $5-15 \mathrm{~mm}^{2}$ etendue range have geometrical efficiencies about $40-50 \%$ for the best condensers; although theory allows about $100 \%$ (no reflection nor absorption losses are considered). The problem is in the coma aberration of the reflectors and the rotational symmetric image of the source making the source projected image to unfit with the target. Thus, the only way to improve this performance is to generate a free form design that is able to control the shape and rotation of the source projected images. As yet, this can only be done with the SMS3D design method. We present here one of such designs achieving a collection efficiency 1.8 times that of an elliptical condenser for a 4:1 target aspect ratio and for the range of target etendue with practical interest and 1.5 for 16:9 target. These designs use only 1 additional reflection, i.e., use a total of 2 reflections from the source to the target. A prototype of one type of free form condenser has already been built.
\end{abstract}

Keywords: Condenser, free form, geometrical optics, optical design, lens design, mirrors

\section{INTRODUCTION}

The main function of the condenser optics in a projection system is to collect as much light as possible from the source and transmit it to the microdisplay, which will module spatially the light towards the projection optics with a high average luminance. A new conceptual design for condenser lenses is presented here. This device has been designed with the SMS method in its 3D version [1]-[7]. A detailed explanation of the SMS3D method has been recently given in [8].

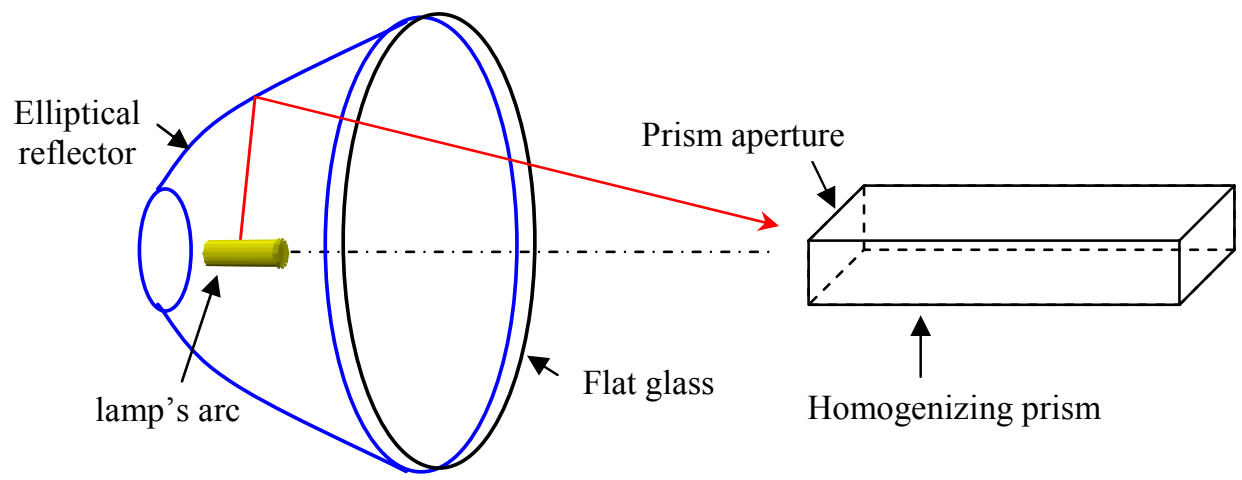

Fig. 1. Conventional condenser with elliptical reflector.

Most conventional condensers use elliptic or parabolic mirrors (see Fig. 1). They perform far from the theoretical limits (calculated using the etendue invariance) for sources such as arc lamps or halogen bulbs. Typical small displays in the 5$15 \mathrm{~mm}^{2}$ srad etendue range have collection efficiencies about $40-50 \%$ for the best condensers, although theory allows about $100 \%$ (collection efficiency defined as the power sent to the target over the source power).

*ic.minano@upm.es ; phone +34 913364507; fax +34 913364501

Illumination Optics, edited by Tina E. Kidger, Stuart R. David

Proc. of SPIE Vol. 7103, 71030A - (c) 2008 SPIE · CCC code: 0277-786X/08/\$18 · doi: 10.1117/12.794259

Proc. of SPIE Vol. 7103 71030A-1 
To understand the limitations of conventional elliptic or parabolic condensers it is useful to consider the concept of the projection of source-images. A pinhole at the exit optical surface of the condenser will let pass a bundle of rays that bear the local image of the source (see Fig. 2). The limited collection efficiency for small etendues of a conventional elliptical condenser can be understood by looking at its projected source images. Projected source-images of conventional condensers have two notable characteristics:

1. The size of the projected image varies (in meridian length $\mathrm{m}$ and sagittal width $\mathrm{s}$ ) from point to point along the profile of the condenser exit aperture. This is because the source-images are about 4 times longer than wide (because they replicate the elongated size of the UHP arc) and because the coma of the elliptical or parabolic reflector.

2. The elongated source-images rotate, due to the rotational symmetry of the mirror and the fixed orientation of the light source. These variously rotated bar-like arc images do not collectively fit well with the usual rectangular targets

The drawings show the arc as a surface-emitting cylinder, which is a schematic simplification of the actual nonhomogeneous luminance distribution of an arc (Fig. 2.b). This simplification helps understand the inherent limitations of the elliptic condenser, because the etendue of the simplified arc is a better defined parameter than that of the real arc.

When the etendue of the target (i.e., of the homogenizing prism and consequently the etendue of the microdisplay) is much greater than that of the source (arc), the two aspects mentioned above (projected image size variation and images rotation) do not limit the collection efficiency, since the homogenizing prism aperture (shown as a dashed-line rectangle in Fig. 2.d) will be much larger than all the projected arc images. This, however, implies the decrease of the average brightness of the source on the target. The consequences of a low average brightness (with a given total flux on the screen) are the use of a large (and expensive) microdisplay and/or the use of an expensive optical projector. In the most cost-demanding applications the microdisplay etendue will be smaller and closer to that of the arc.

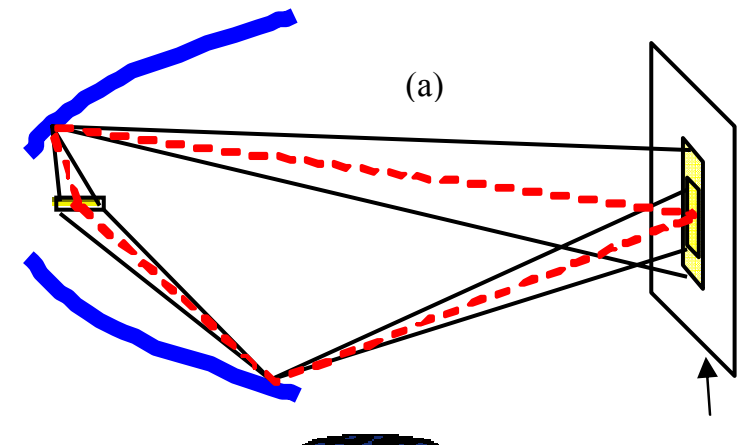

(b)

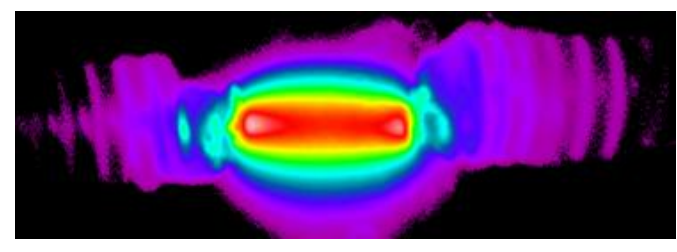

Target plane (where homogenising prism entrance is placed)

(c)
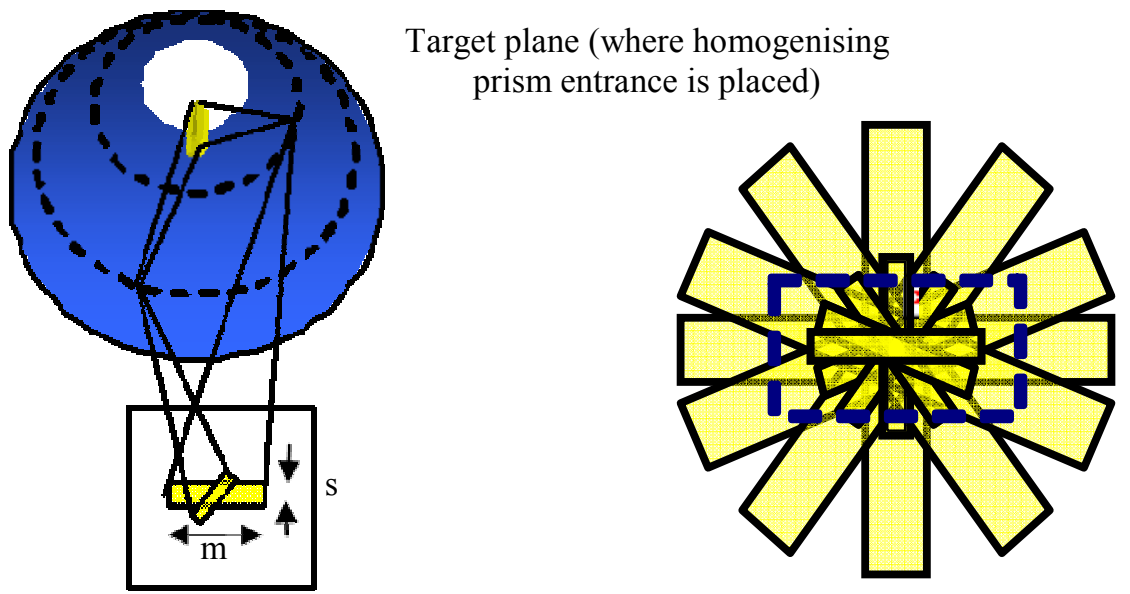

Fig. 2. Notable characteristics of the source images of any conventional elliptical condenser. (a) variable lengths, (b) as they replicate the elongated shape of the UHP arc, they are about 4 times longer than wide, (c) they rotate at the target due to the condenser's rotational symmetry, (d) Source images do not fit well with the panoramic 16:9 target, shown as a dashed-line rectangle.

An interesting clarifying case is that in which both microdisplay and arc etendues are equal. There is not theoretical obstacle for a $100 \%$ collection efficiency although no such design is known. It can be shown that in this equi-etendue case the homogenizing prism entry aperture will have the same area as the average projected image. This makes clear 
that the variable size and the rotation of the images of the elliptical condenser do prohibit $100 \%$ collection efficiency. A condenser achieving such $100 \%$ value would necessarily meet the condition that all projected images are rectangular and exactly match the contour of the prism entry aperture. This condition is general, i.e., it does not depend on the optical elements used in such a hypothetical $100 \%$ collection condenser.

A recent trend in improving collection efficiency for small etendues has been to reduce the arc etendue by reducing the gap between the electrodes. Furthermore, some optical designs have being developed to improve the collection efficiency by:

1. Reducing the arc etendue via a hemispherical mirror (concentric with the arc) which reflects half of the emitted light back to the arc (this one is partially absorbent) and through it increasing its luminance [9], [10]. The halfetendue light from the hemisphere not covered by the mirror arc is then collected by a conventional elliptical reflector.

2. Creating a side-by-side image of the arc with a decentered hemispherical mirror, and creating a composite 1:1 image of that via a dual parabolic reflector [11].

3. The equalization of the meridian length of the projected arc images [12], [13] by correcting the elliptical mirror coma, using an aspheric reflector profile and an aspheric lens, both surfaces still being rotationally symmetric.

These optical approaches, apart from their complexity and technological challenges, have limited gain capability because their condenser optics is still restricted to being rotationally symmetric.

Other approaches to improve system efficiency include color recapture [14] and color scrolling [15] (which try to recover the losses produced by the color filtering in single microdisplay projectors), or polarization-recovery techniques [16] (which try to recover the 50\% losses produced by the need of polarized light in LCD and LCoS systems). In these approaches, however, the resulting lamp etendue is accordingly increased (doubled and tripled in polarization and color recovery systems, respectively), further limiting the performance of small microdisplays that use classical condensers.

Regarding the state of the art of manufacturing condenser optics, all present systems are based exclusively on rotationalsymmetric surfaces. These are manufactured mainly from glass (due to its low cost) or by glass-ceramic (for higher thermal stability). The accuracy of both techniques is limited, so the manufactured profiles can differ substantially from those intended.

\section{DESIGN OF THE XX CONDENSER}

The simplest application of the SMS3D design method provides full control of two couples of wavefronts and the partial control of a third one with just two optical surfaces (mirrors or dioptrics). Thus the size, position, and orientation of the projected source-images can be controlled to an unprecedented level. SMS-designed dual reflector devices are herein termed XX. A more detailed explanation of the design procedure of the XX condenser is given in [17].

The XX configuration and geometry given in [17] can also be applied for the problem of coupling the rays issuing a cylindrical source into a rectangular target. This is of practical interest in condenser applications, because the resulting design, as shown below, can efficiently couple the light from an arc into a rectangular aperture. The SMS3D's control of the projected source images (via the control of selected wavefronts) allows the non-rotational projection of them, and the constancy of projected size which is perfect in at least one dimension, and guaranteed around a curve of the mirrors (called seed rib) in the perpendicular dimension) (see Fig. 3). This perfect control of one dimension of the projected size and partial control of the other dimension results to be enough for our purposes.
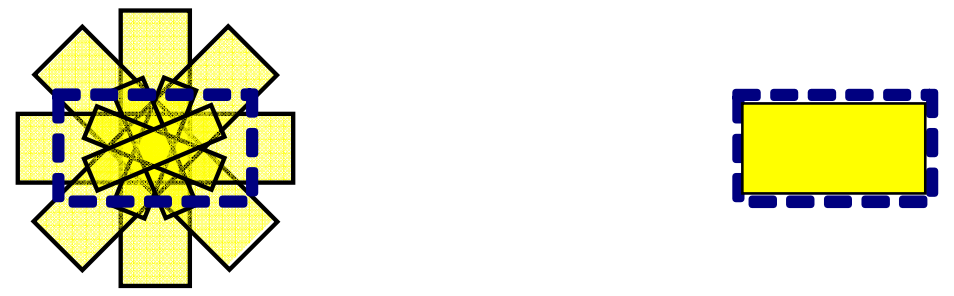

Fig. 3. The XX 3D condenser, in contrast to conventional condensers (left), can be designed to avoid the rotation of projected arc images so that it can fit rectangular apertures.

The formal definition of this problem is (see Fig. 4): The rays of the source have all the same radiance and are those emitted from a cylinder surface whose directions form an angle greater than $\beta_{\text {MIN }}$ with the cylinder's axis. The rays 
accepted by the target are the ones reaching a rectangle forming an angle smaller than $\phi_{\mathrm{MAX}}$ with the normal to the rectangle. The condenser has to maximize the power transferred from the source to the target.
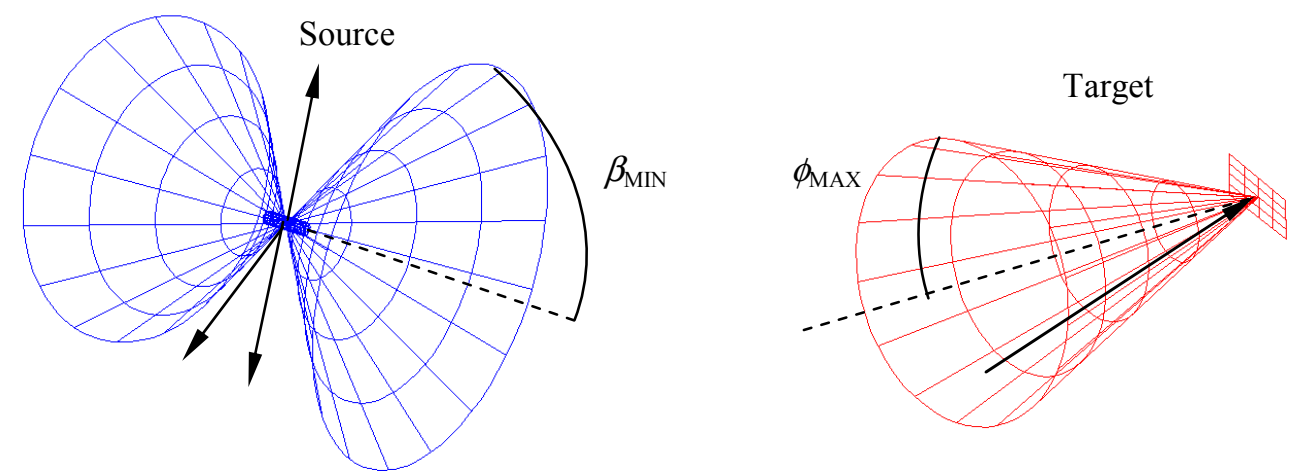

Fig. 4. Source and target definitions. Any of the points of the source (a cylindrical surface) emits in its entire open hemisphere excepting in the directions forming an angle smaller than $\theta_{\mathrm{p}}$ with the cylinder axis. The target is a rectangle accepting radiation coming within a cone normal to the target surface.

The input and output wavefronts used in the SMS 3D design are indicated in Fig. 5. All of them belong to the edge rays of the bundles defined for the source and the target. All of the output wavefronts $\mathrm{WF}_{\mathrm{ox}}$ are spherical and centered at the midpoints of the rectangle sides of the target. Two of the wavefront couples $\mathrm{WF}_{\mathrm{i} 3}-\mathrm{WF}_{\mathrm{o} 3}$ and $\mathrm{WF}_{\mathrm{i} 4}-\mathrm{WF}_{\mathrm{o} 4}$ are used for the calculations of the SMS chains while the other two couples $\mathrm{WF}_{\mathrm{i} 1}-\mathrm{WF}_{\mathrm{o} 1}$ and and $\mathrm{WF}_{\mathrm{i} 2}-\mathrm{WF}_{\mathrm{o} 2}$ are only used for initial curve calculation. This is why there is only a partial control of one dimension of the source images. $\mathrm{WF}_{\mathrm{i} 3}$ and $\mathrm{WF}_{\mathrm{i} 4}$ are orthonormal rays issuing form the cylinder edges. $\mathrm{WF}_{\mathrm{i} 1}$ and $\mathrm{WF}_{\mathrm{i} 2}$ are formed by rays that are tangent to the cylinder.

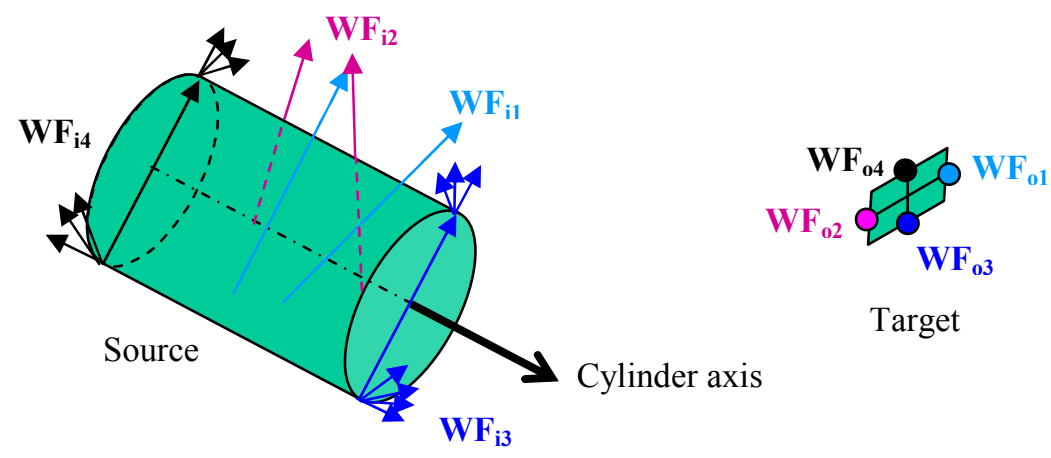

Fig. 5. Rays of the input wave fronts $\left(\mathrm{WF}_{\mathrm{ix}}\right)$ and center points of the output wavefronts $\left(\mathrm{WF}_{\mathrm{ox}}\right)$ used for the SMS 3D design.

Four families of solutions were defined according to the signs of the magnifications related with each pair of wavefront couple. These families are more detailed analyzed in [17]. There is still another Boolean variable to be added to the signs of the magnifications, raising the four families of XX solutions to eight). This third Boolean variable appears from the additional possibility of choosing that the half of the POE mirror at $y>0$ reflects the light towards the half of the SOE at $\mathrm{y}>0$ (adjacent reflectors) or towards to half of the SOE at $\mathrm{y}<0$ (non adjacent).

\section{RAY TRACING RESULTS.}

In this section, only the case with the best performance (among the aforementioned families of this XX condensers) is shown. This case has negative magnifications, with not adjacent POE and SOE paired halves. The input parameters of this design are:

1. Cylindrical source: Length $\mathrm{L}=1.2 \mathrm{~mm}$; Diameter $\mathrm{D}=0.3 \mathrm{~mm} ; \beta_{\mathrm{MIN}}=45^{\circ}$.

2. Rectangular flat target: Aspect ratio $=4: 1 ; \phi_{\mathrm{MAX}}=19^{\circ}$. 
The distance from source center to target plane was fixed to $30 \mathrm{~mm}$. Fig. 6 shows the surfaces of the mirrors of the XX design, while Fig. 7 shows the standard top, side and front views, indicating the dimensions.
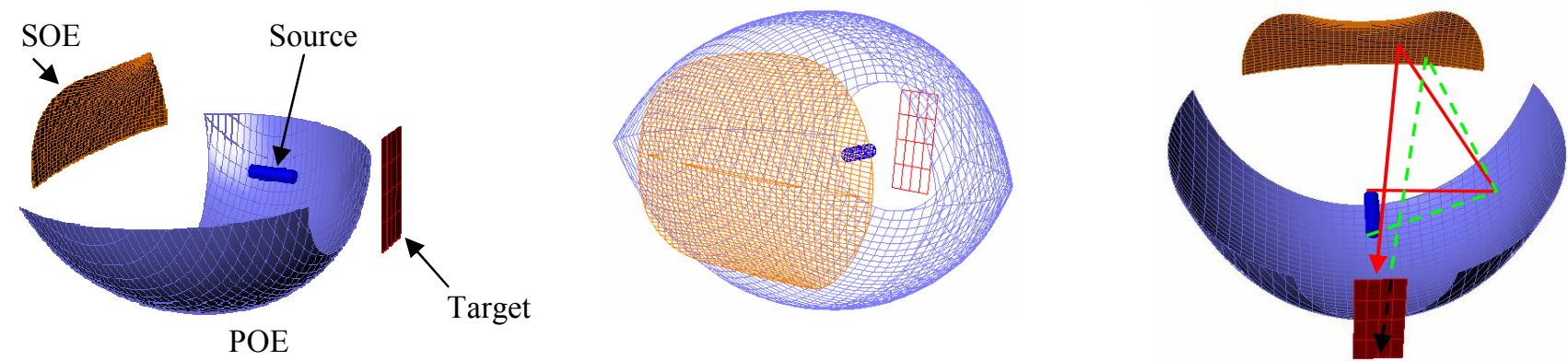

Fig. 6. Selected $\mathrm{XX}$ design of the family $\mathrm{M}<0, \mathrm{~N}<0$ and non-adjacent $\mathrm{POE}$ and $\mathrm{SOE}$ paired halves (only POE with $\mathrm{y}<0$ and SOE with $y>0$ are shown at the top). Source and targets are not shown to scale.
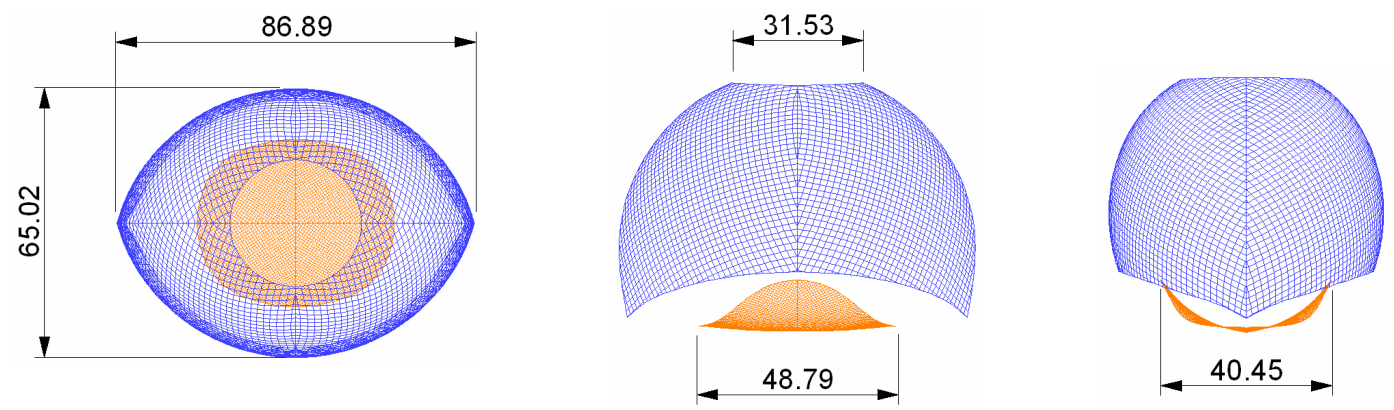

Fig. 7. Some dimensions (in $\mathrm{mm}$ ) of the selected XX design of Fig. 6 (in mm).

In order to evaluate performance, the collection efficiency versus target etendue has been calculated by ray-tracing (using the commercial ray-tracing package LightTools ${ }^{\circledR}$, see www.opticalres.com) and it is shown in Fig. 8 versus the etendue of the target. This etendue is given by $E_{\text {target }}=A_{\text {target }} \pi \sin ^{2}\left(\phi_{\mathrm{MAX}}\right)$, where $A_{\text {target }}$ is the target area and $\phi_{\mathrm{MAX}}= \pm 19^{\circ}$. The etendue of the target has been varied by varying the target area $A_{\text {target }}$ while freezing the target aspect ratio (4:1) and its circular field of view $\phi_{\mathrm{MAX}}$.

For comparison purposes, Fig. 8 shows two further curves. One of them is collection efficiency versus target etendue for a conventional elliptical reflector working for the same source and a target with the same aspect ratio (4:1). The eccentricity of the ellipsoid has been set equal to 0.8 (which is the optimized standard) and the target field of view of $\phi_{\mathrm{MAX}}= \pm 30^{\circ}$ (which is also the standard value in the market).

The third curve in Fig. 8 corresponds to the theoretical limit, which is deduced by etendue considerations: an ideal condenser achieving it (which may not exist) would transfer all the source power to the target if the target etendue is greater than the source etendue (i.e., it will have will have a $100 \%$ collection efficiency in this case), and would fully fill the target etendue with light from the source if the source etendue is larger than the target etendue. Then the ideal condenser will have a collection efficiency equal to the ratio of target to source etendue when the target etendue is smaller than the source etendue. 

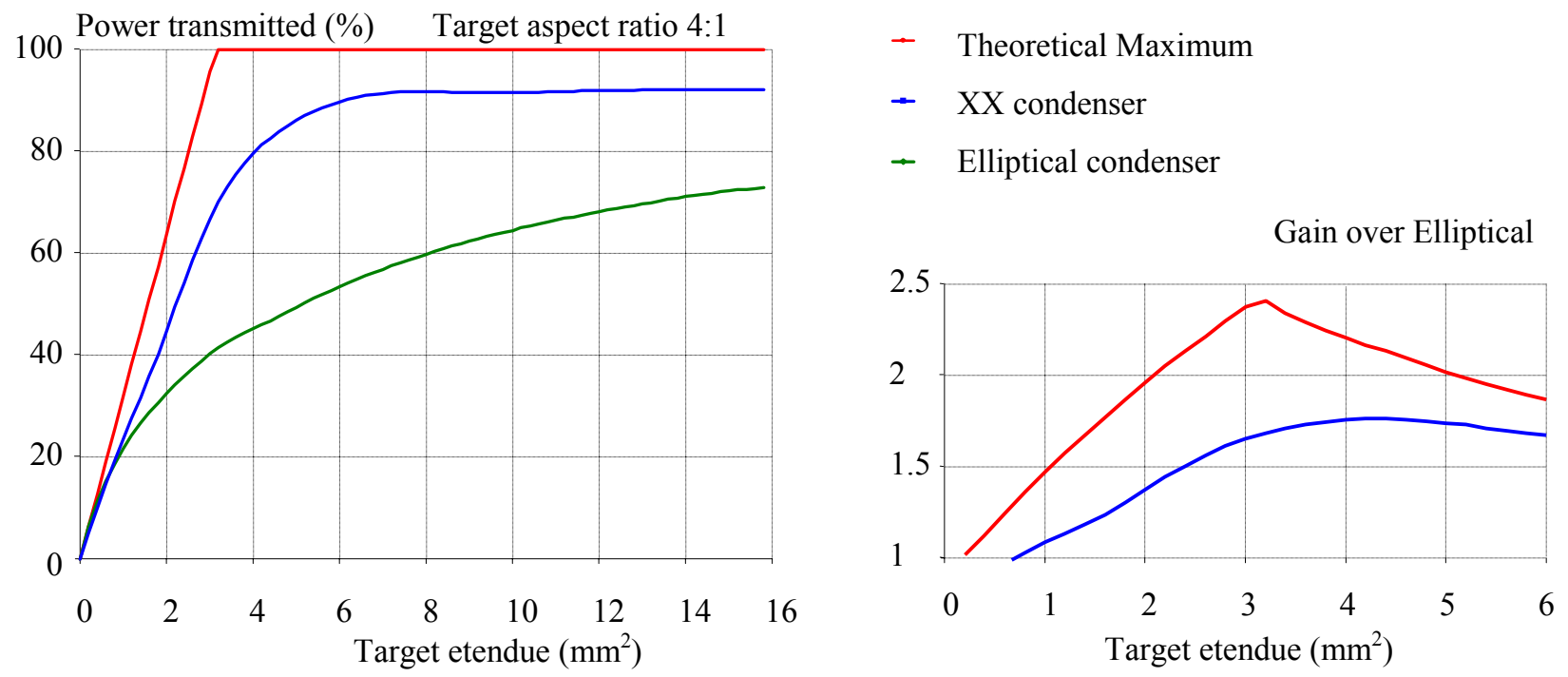

Fig. 8. Ray tracing results: collection efficiency versus the 3D etendue of the target for the condenser in Fig. 6 . The maximum theoretical performance and that of conventional elliptical condenser are also shown for comparison. All mirrors have $100 \%$ reflectivity. The 3D etendue of the source is $3.13 \mathrm{~mm}^{2}$. Gain defined as the ratio of the collection efficiencies of a given non-conventional condenser to that of the elliptical condenser. XX in Fig. 6 shows gains up to 1.8 in a wide range of etendue. The theoretical limit could achieve gains greater than 2.4.

The etendue of the source can be calculated with the cylindrical source geometry:

$$
E_{\text {source }}=\pi D L\left(\pi+\sin \left(2 \beta_{\text {MIN }}\right)-2 \beta_{\text {MIN }}\right)+\frac{1}{2} \pi^{2} D^{2}\left(1-\sin ^{2} \beta_{\text {MIN }}\right)
$$

For the above input data $\mathrm{E}_{\text {source }}=3.13 \mathrm{~mm}^{2}$. Fig. 8 and shows that the XX performs much better than the elliptical reflector (for all mirrors, specular reflectivity has been set equal to 1), getting close to the theoretical limit. There are three factors preventing the XX from reaching the theoretical limit:

1. When target etendue is large some rays that reflect off the POE will miss the SOE, so that XX curve cannot reach $100 \%$ collection efficiency.

2. The "shoulder" of the XX efficiency curve is rounded (in contrast to the theoretical one, which shows a slope discontinuity). This is due to the non-stepped transitions of the illuminance distribution on the target plane and also to the rounded contour lines of that illuminance distribution (shown in Fig. 9).

3. When target etendue is small, the slope of the XX curve in Fig. 8 is less than the theoretical slope, because the $\mathrm{XX}$ does not fill completely and uniformly the circular field of view (see the intensity distribution in Fig. 10). 


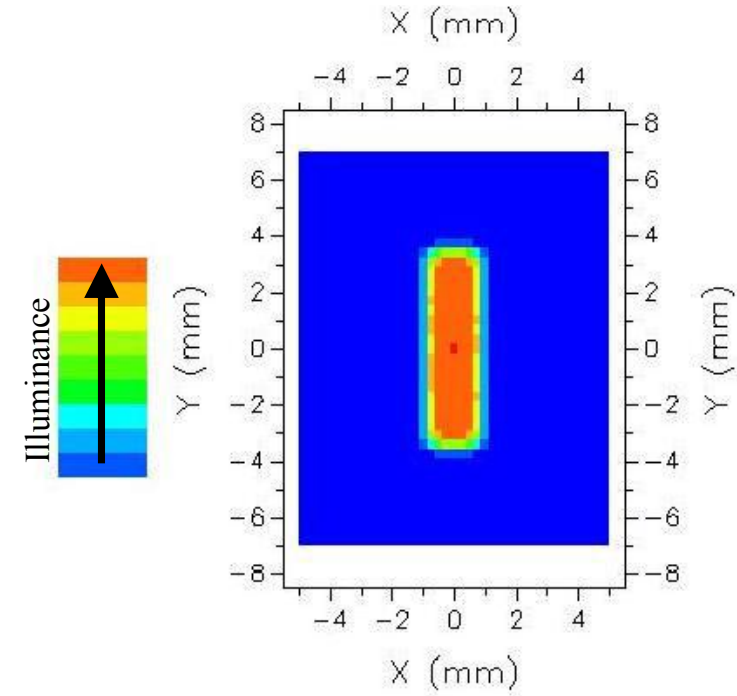

Illuminance

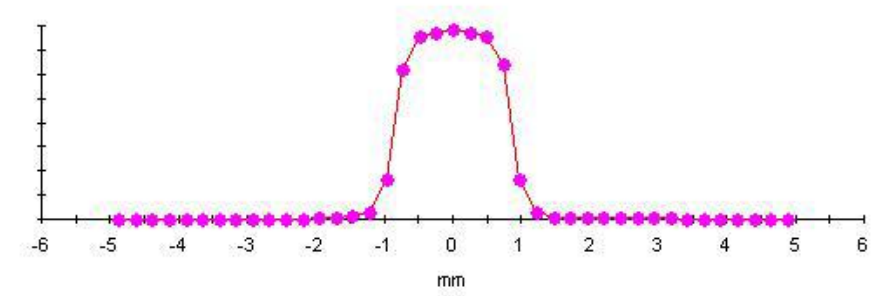

Current $\times$ Slice $(\mathrm{mm})$

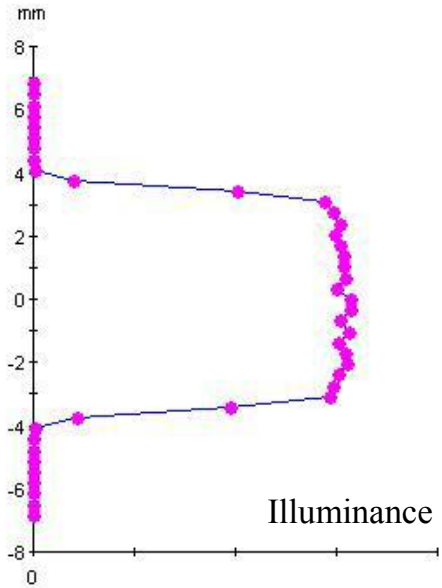

Current $Y$ Slice $(\mathrm{mm})$

Fig. 9. Ray tracing results: illuminance distribution on the target plane for the condenser in Fig. 6,.
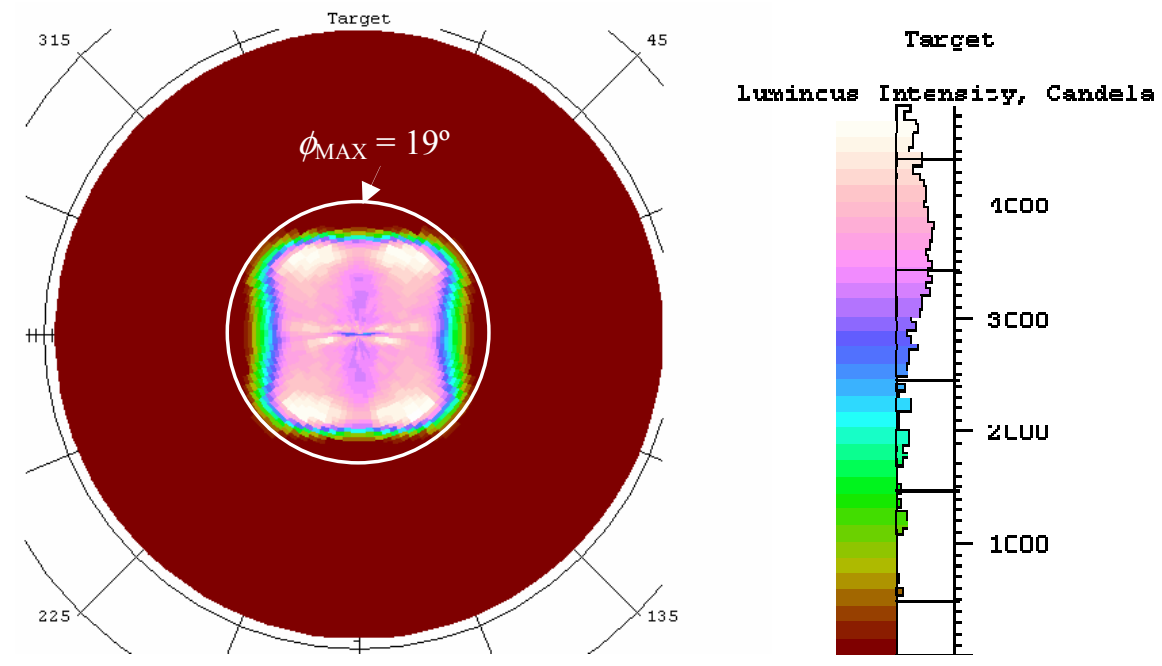

Fig. 10. Ray tracing results: intensity distribution through the target plane for the condenser in Fig. 6, assuming the source has a flux of $1000 \mathrm{~lm}$.

The 4:1 aspect ratio of the target is not seen in projection display applications. On the contrary, the 16:9 format can be considered the present standard. An XX condenser similar to that in Fig. 6, designed for a cylindrical source with 
diameter $0.62 \mathrm{~mm}$ (source etendue $=6.96 \mathrm{~mm}^{2}$ ) and keeping the rest of parameters unchanged, will produce a 16:9 irradiance distribution on the target plane. Fig. 11 shows the ray tracing results. The XX with target's circular field of view of $\phi_{\mathrm{MAX}}=19^{\circ}$ still performs better than elliptical reflector, although the gain is reduced to 1.5 , due to the lower aspect ratio of the target (again, for all mirrors, specular reflectivity has been set equal to 1). The theoretical limit is also reduced to 2 .

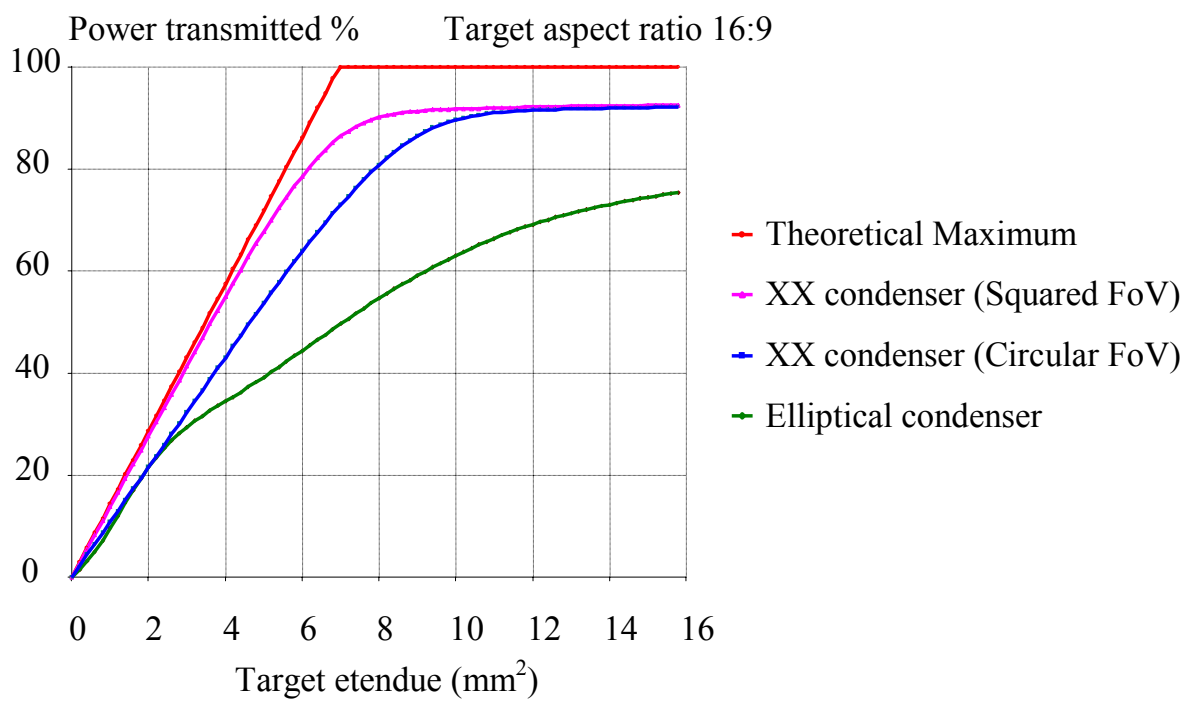

Fig. 11. Ray tracing results: collection efficiency of an XX condenser versus the 3D etendue of a 16:9 target with circular and square Field of View (FoV). The maximum theoretical performance and the conventional elliptical condenser (with circular FoV) are also shown for comparison. All mirrors have $100 \%$ reflectivity. The 3D etendue of the source is 6.96 $\mathrm{mm}^{2}$.

It is interesting to note that these XX condensers perform closer to the theoretical limit if a square field of view for the target is considered. Fig. 11 also shows the collection efficiency and target etendue using a square field of view of $28^{\circ} \times 28^{\circ}$ for the XX at the target (this square field of view is almost inscribed in the preceding $19^{\circ}$ radius circular filed of view). This result is more than just an academic consideration if we taken into account that the angular acceptance field of the dielectric filled mixing prism working with total internal reflection has a squared shape. The theoretical limit stays unchanged but the XX performs much better because its intensity distribution matches better with the square field of view. Note that the slope of the efficiency curve of the XX with square field of view becomes now very close to the theoretical limit near the origin, indicating a uniform and well-filled field of view.

\section{DEMONSTRATOR PROTOTYPE}

A demonstrator prototype has been fabricated to prove these design principles. For easier handling, instead of a projector arc lamp (whose high flux cannot be dimmed; it also has high UV emission), an automotive $\mathrm{H} 7$ halogen lamp was selected. The $\mathrm{H} 7$ filament is a spiral enclosed by a cylinder of length $\mathrm{L}=4.3 \mathrm{~mm}$ and diameter $\mathrm{D}=1.55 \mathrm{~mm}$. The lamp geometry conditioned the choice of the XX configuration, to avoid shading and mirror to lamp interferences. The design selected is an $\mathrm{XX}$ of the family $\mathrm{N}<0, \mathrm{M}<0$ with adjacent $\mathrm{POE}$ and $\mathrm{SOE}$ halves. Moreover each half has a separate rectangular target, as shown in Fig. 12. Note that the 3D drawing in Fig. 12.b shows the device rotated 90 degrees around the cylindrical source axis, in contrast to the device in Fig. 6.

The prototype, manufactured by nickel electroforming, is shown in Fig. 13 and Fig. 14. The mirror coating is made of evaporated aluminium. The $y>0$ and $y<0$ halves of the SOE mirror were made as (identical) replicas of the mold. Similarly, the POE was made of two halves, but in this case the POE was split into $x>0$ and $x<0$ halves, for easier demolding. Fig. 13 (a) shows the whole condenser. The upper POE has bee taken out in Fig. 13 (b) showing the lamp and the SOE. 

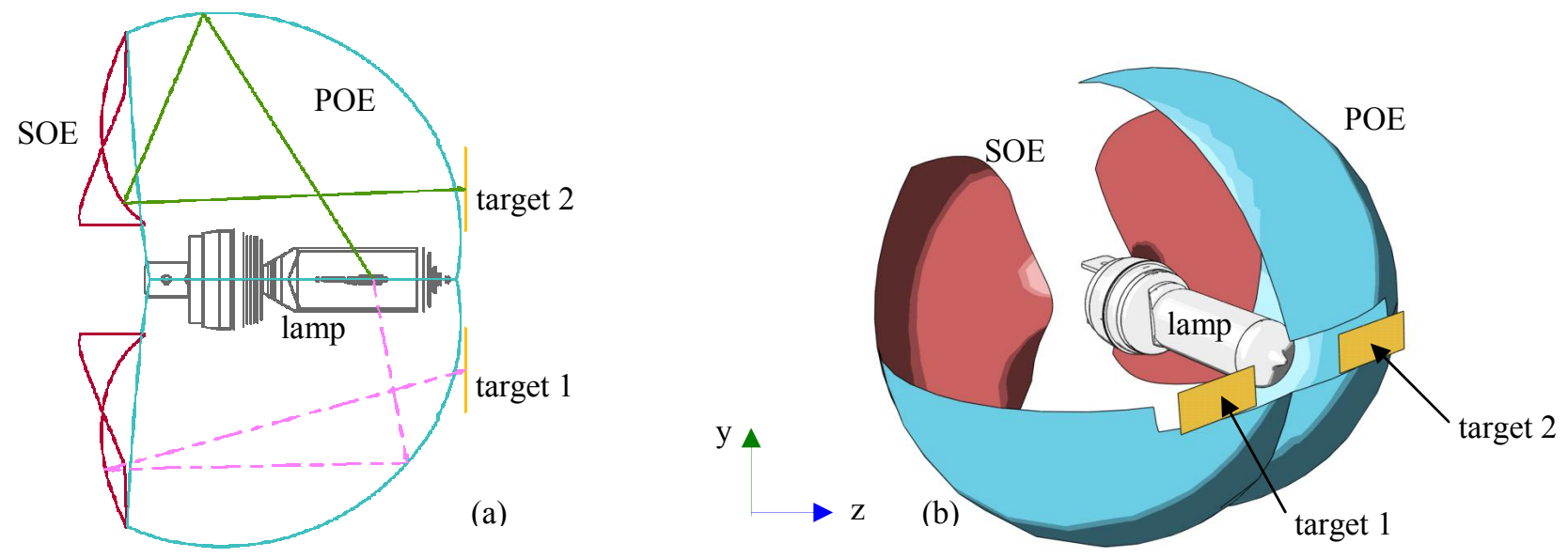

Fig. 12. Demonstrator prototype diagrams.

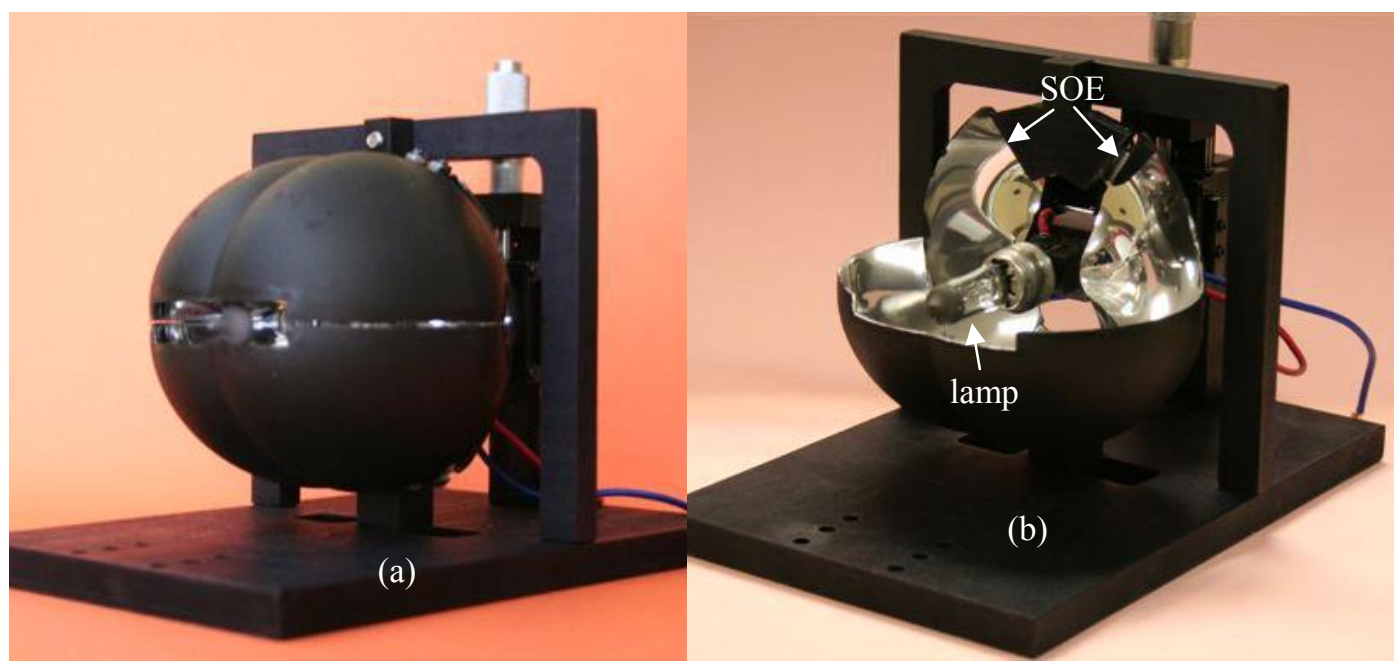

Fig. 13 Nickel electroformed XX condenser demonstrator for a filament lamp and two targets. One POE reflector half has been removed in (b) to show the lamp and the SOE.
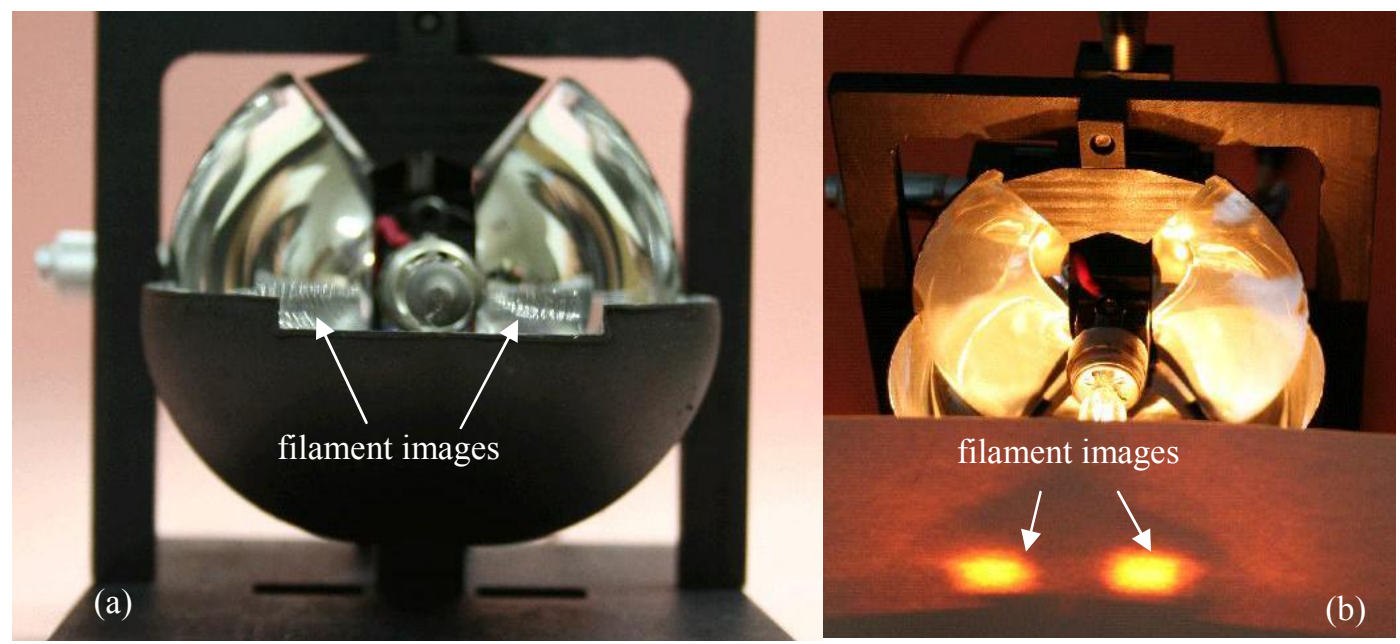

filament images

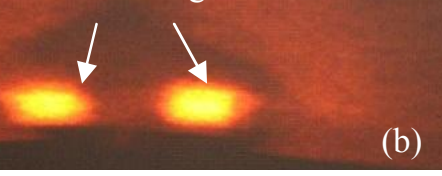

Fig. 14. XX condenser demonstrator. The upper POE reflector half has been removed. The photography in (a) is taken within the angular field of the targets so the filament images can been seen. When the filament is emitting, the two images are formed on a paper placed at the target's plane (b). 
The photography of Fig. 14(a) has been taken within the angular field of the target. It shows the two images of the filament formed at the exit plane. Fig. 14(b) shows the two spots formed by the condenser on a paper placed at the condenser target plane. For a clearer understanding of the arrangement, the upper POE has been taken out form Fig. 14(a) and (b). No radiometric measurements have been done yet with this prototype.

\section{CONCLUSIONS}

After introducing the efficiency limitations of some conventional condensers, we have presented new condenser configurations based on an XX 3D design. These new condensers have capabilities unattainable with rotational symmetric optics, particularly the control of the rotation of the projected source images. These condensers are designed with the SMS3D design method. The condensers use two reflectors. Ray traces have been done using a cylindrical source emitting with constant radiance within a limited angular field and a rectangular target with a circular cone angular field. Using the same source type and target we have also ray traced a conventional elliptical condenser. A comparison of Power Transmitted vs Target Etendue (done under equal conditions) shows that one type of XX condenser can send to the target up to 1.8 times the power sent by the elliptical condenser (for a 4:1 aspect ratio target rectangular aperture) and up to 1.5 times when the target aperture aspect ratio is 16:9. These XX designs have shown the powerful capabilities of the SMS3D design method to handle free-form optical surfaces.

Although it was known long time ago that the rotational symmetry was limiting the capabilities of condensers, this is the first time (up to our knowledge) that a free-form condenser design outperforming a rotational symmetric one is shown. This is an important theoretical achievement showing that the extra degrees of freedom provided by free-form surfaces can be used to improve the performance of the design. Nevertheless there is still a lot of work to be done from the practical point of view. The main drawback of the XX condenser is the use of two mirrors instead of the single one used by the conventional elliptical condenser. The extra reflection makes the XX condenser less tolerant to errors, not only surface errors in the mirrors but also the errors on the position of the source which could be the dominant ones in a mass production assembling procedure using arc lamps.

An XX condenser demonstrator has been built by electroformed nickel to show the concept. For easy handling the prototype, we have chosen a lamp filament as source. As yet no radiometric measurement has been done with it.

\section{ACKNOWLEDGEMENT}

The authors thank the Spanish Ministry Mityc (Foco 1000x: FIT-330100-2007-49 and PRV: FIT-330101-2007-3), and the Madrid Regional Government (CCG07-UPM/000-1602 and CCG07-UPM/ENE-1603) for the support given in the preparation of the present work. The authors from the Universidad Politécnica de Madrid also thank Optical Research Associates for the educational license of LightTools software.

\section{REFERENCES}

[1] J.C. Miñano, J.C. González, "New method of design of nonimaging concentrators", Appl. Opt. 31, pp. 3051-3060, (1992)

[2] J.C. Miñano, P. Benítez, J.C. González, "RX: a nonimaging concentrator", Appl. Opt. 34, pp. 2226-2235, (1995)

[3] J.C. Miñano, J.C. González, P. Benítez, "A high-gain, compact, nonimaging concentrator: RXI", Appl. Opt. 34, pp. 7850-7856, (1995)

[4] P. Benítez, J.C. Miñano, "Ultra high numerical aperture imaging concentrator". J. Opt. Soc. of Am., 14, 8 (1997), pp. 1988-1997.

[5] R. Winston, J.C. Miñano, P. Benítez, Nonimaging Optics, (Academic Press, New York, 2005)

[6] W. Cassarly, "Nonimaging Optics", in Handbook of Optics, 2nd ed., pp 2.23-2.42, (McGraw-Hill, NewYork, 2001).

[7] P. Benítez, J.C. Miñano, J. Blen, R. Mohedano, J. Chaves, O. Dross, M. Hernández, J.L. Alvarez, W. Falicoff. "SMS Design Method in 3D Geometry: Examples and Applications" Nonimaging Optics: Maximum Efficiency Light Transfer VII, R. Winston ed.. Procc. of SPIE Vol 5185., pp 18-29.(2003)

[8] J. Chaves, Introduction to Nonimaging Optics, (CRC Press, Boca Ratón, 2008).

[9] H. Moench and A. Ritz. "Higher Output, More Compact UHP Lamp Systems", SID Int. Symp. Digest Tech. Papers (33)1, 1160-1163 (2002). 
[10] US Patent $6,356,700$ by Strobl (2002)

[11] US Patent $6,672,740$ by Li (2004)

[12] JP Patent $7,174,974$ by Tadaaki (1995)

[13] US Patent 5,966,250 by Shimizu (1999)

[14] D.S. Dewald, S.M. Penn, M. Davis, "Sequential Color Recapture and Dynamic Filtering: A Method of Scrolling Color", SID Int. Symp. Digest Tech. Papers (32)1, 1076-1079 (2001)

[15] J.A. Shimizu, "Scrolling Color LCOS for HDTV Rear projection", SID Int. Symp. Digest Tech. Papers, (32)1, 10721075 (2001)

[16] M. Duelli, A.T. Taylor, "Novel polarization conversion and integration system for projection displays", SID Int. Symp. Digest Tech. Papers (34)1, 766-769 (2003).

[17] J.C. Miñano, P. Benítez, J. Blen, A. Santamaría "Design of a Novel Free-Form Condenser overcoming rotational symmetry limitations" Novel Optical Systems Design and Optimization XI, Proc. SPIE 7061A, San Diego 2008. 